\title{
Preferred Position of the Detector for MeV Backscattering Spectrometry
}

\author{
F.H. Eisen and M.-A. Nicolet \\ California Institute of Technology, Pasadena, CA 91125
}

\begin{abstract}
When an amorphous target is tilted with respect to the incident beam, the yield of an MeV backscattering spectrum will not change height if the measurements are executed under suitable conditions. One of these involves the position of the detector with respect to the plane defined by the directions of the incident beam and the tilt axis, as demonstrated here. The results clarify early data on the subject.
\end{abstract}

Statistical-mechanical arguments going back to Lindhard [1] lead to a rule of averages that applies to nuclear encounters as they occur in $\mathrm{MeV}$ backscattering processes. Specifically, the rule says that the rate of interactions averaged over solid angle is the same in single crystalline and in amorphous targets when the energy loss mechanisms are negligible. The rule applies for averages over $4 \pi$ but it may be valid for averages over smaller angles as well [2]. That rule is invoked when simulating the backscattering spectrum of an amorphous target with a single crystalline target. The incident beam impinges on the single crystal sample with a tilt angle, $\varphi$, that is well outside the critical angle of an axial channel, and the target is rotated around that channeling axis of the crystal. In so doing the incident beam describes a cone around the channeling axis and passes through planar channels associated with that axis. The shoulders and dips in the scattering yield cancel, resulting in a backscattering spectrum that equals the yield of a target with a random arrangement of atoms.

We have submitted this assertion to an experimental test by comparing the backscattering spectra of a 0.7 to $3.0 \mathrm{MeV}^{4} \mathrm{He}$ beam impinging on a polycrystalline silicon target, on an amorphized silicon target, and on silicon single crystalline targets of $<111>,<110>,<100>$ orientation tilted by fixed angles of $5^{\circ}<\varphi<15^{\circ}$ with respect to the incident beam and rotated during the acquisition of the spectra [3]. All spectra were practically indistinguishable, which indicates that within the accuracy of the measurements, Lindhard's rule does apply for these experiments. We did find, however, that care has to be devoted to several experimental details, of which one is discussed here. Consider the arrangement of an incident beam, a planar target holder, and a detector as depicted in Fig.1.

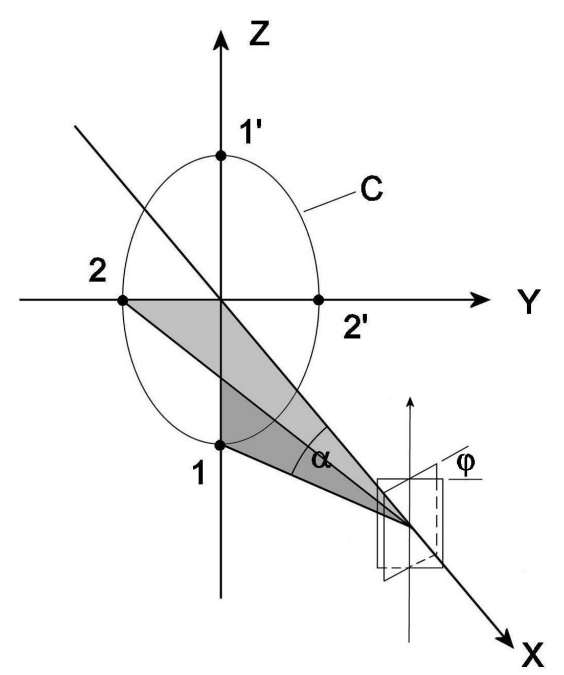

FIGURE 1. To detect particles of a beam incident along the $\mathrm{x}$-axis and backscattered from a target by the scattering angle $180^{\circ}-\alpha$, any position on the circle $C$ is acceptable for the detector. When the target is tilted by the angle $\varphi$, only the detector positions 1 and 1 ' leave the height of the backscattering yield unchanged. For positive values of $\varphi$ the yield rises; for negative values of $\varphi$ it decreases.

The target holder has two axes that are perpendicular to each other. Motion about one axis rotates the 
sample holder in its own plane. This axis is collinear with the incident beam when the tilt about the other axis is set to $\varphi=0$. In Fig. 1 the beam is arbitrarily chosen to run horizontally in the $\mathrm{x}$-direction while the tilt axis is arbitrarily assumed to be vertical and parallel to the $\mathrm{z}$-direction of an orthogonal coordinate system. The beam and the tilt axis define the $x-z$ plane. As the plane of the target holder is tilted by the angle $\varphi$, the rotation axis sweeps in the $x-y$ plane.

For a flat target and a chosen backscattering angle $\alpha>0$ the detector may be positioned anywhere on the cone formed by that angle and whose apex is at the beam spot on the target (Fig.1) as long as the holder is perpendicular to the beam $(\varphi=0)$. The situation is different when the sample is tilted. When the detector is in the plane of the beam and the tilt axis (positions 1 and 1' in Fig.1) a backscattered particle that is detected with a fixed energy $E_{1}$ follows the same inward and outward paths regardless of tilt. For all other detector positions the total path length is a function of the tilt angle. Figure 2 shows the paths, viewed from above, for particles scattered in a flat target from the same depth $\mathrm{d}$ (solid lines) for two target positions with $\varphi=0$ and $\varphi>0$. For a positive tilt $(\varphi>0)$ the outward path

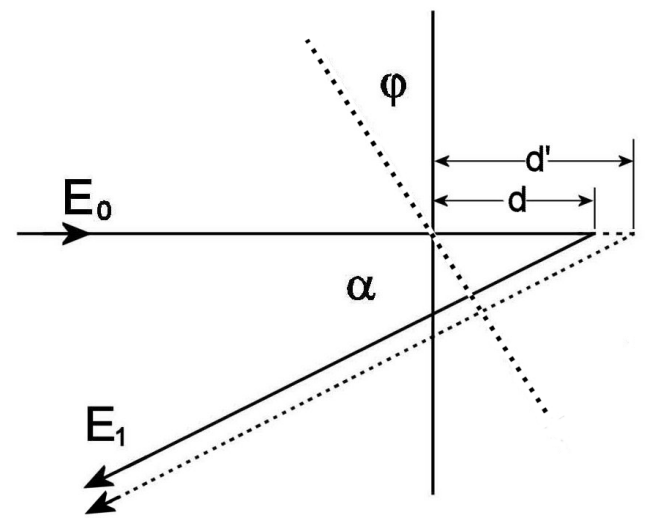

FIGURE 2. Top view of two paths of particles scattered in a flat target and detected at the same exit energy $E_{1}$ when the sample is not tilted $(\varphi=0)$ and when it is tilted by an angle $\varphi$ $>0$, for the detector located in position 2 .

is shortened as a result of the tilting, and the detected energy $E_{1}$ rises. In order for the scattered He to exit the sample with unchanged energy the scattering event must occur at a greater depth $\mathrm{d}^{\prime}$ (dashed lines). This means that the number of scattering events recorded in a fixed energy interval, such as the counts registered in the width $\delta \mathrm{E}$ of one channel in the multichannel analyzer, rises accordingly. Tilting the sample holder by a positive angle $\varphi$ thus increases the yield; a negative tilt reduces it. For position 2 of the detector the ratio of the two depths, $d^{\prime} / d$, is given by

$$
\frac{\mathrm{d}^{\prime}}{\mathrm{d}}=\frac{1+\frac{1}{\mathrm{rK}_{\mathrm{M}} \cos \alpha}}{1+\frac{\cos \varphi}{\mathrm{rK}_{\mathrm{M}} \cos (\alpha-\varphi)}}=\frac{\mathrm{Y}^{\prime}}{\mathrm{Y}}
$$

where $\mathrm{r}=\varepsilon_{\text {in }} / \varepsilon_{\text {out }}$ is the ratio of the average stopping cross sections along the inward and outward paths of the particle [4] and $K_{M}$ is the kinematic factor for the scattering collision [4]. The increase of the backscattering yield $\mathrm{Y}$ of an amorphous target as a function of $\alpha$ is given by that same expression. Figure 3 plots that ratio as a function of the tilt angle $\varphi$ for ${ }^{4} \mathrm{He}$ particles of $2.5 \mathrm{MeV}$, an amorphous silicon target, a scattering angle of $180^{\circ}-\alpha$, and $\alpha=10^{\circ}, 15^{\circ}$ and $20^{\circ}$ with values of $\mathrm{rK}_{\mathrm{M}}=0.425,0.428$, and 0.433 respectively. Figure 4 shows the energy dependence of that ratio for $\alpha=20^{\circ}$ and $\varphi=10^{\circ}, 15^{\circ}$. and $20^{\circ}$.

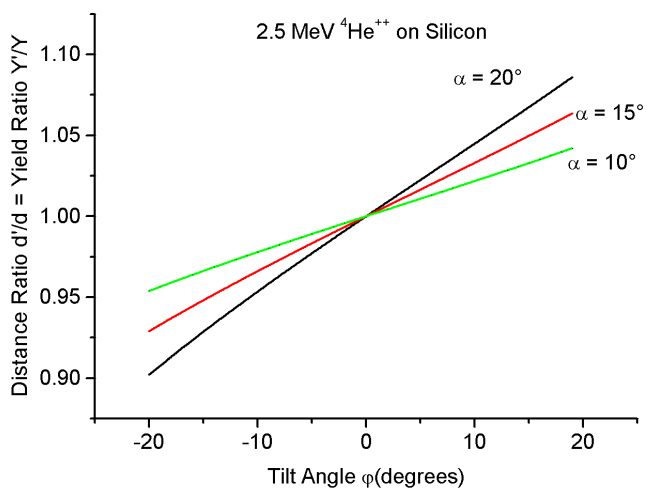

FIGURE 3. The ratio of the penetration depths $\mathrm{d}^{\prime} / \mathrm{d}$ of 2.5 $\mathrm{MeV}{ }^{4} \mathrm{He}$ particles detected with the same exit energy plotted as a function of the tilt angle $-20^{\circ}<\varphi<+20^{\circ}$ and scattering angles of $160^{\circ}, 165^{\circ}$, and $170^{\circ}\left(\alpha=20^{\circ}, 15^{\circ}\right.$, and $10^{\circ}$ ). The target is assumed to be an amorphous silicon wafer. The ratio $\mathrm{d}^{\prime} / \mathrm{d}$ is also that of the backscattering yields $\mathrm{Y}^{\prime} / \mathrm{Y}$ of the backscattering spectrum.

Ziegler and Crowder [5,6] have recorded the 2.5 $\mathrm{MeV}{ }^{4} \mathrm{He}$ backscattering spectra of a $<111>$-oriented silicon wafer amorphized over its first $0.45 \mu \mathrm{m}$ by a silicon irradiation for tilt angles $\varphi$ of $5^{\circ}, 10^{\circ}$, and $15^{\circ}$ and a scattering angle of $160^{\circ}\left(\alpha=20^{\circ}\right)$. They find that the measured backscattering yield must be 
corrected by $-3.3 \%,-6.7 \%$, and $-8.5 \%$ to agree with the yield they observe at $\varphi=0^{\circ}$. Table 1 compares these experimentally determined corrections with those calculated by Eq. 1 under the assumption that the experimental data reported were measured with the detector in position 2 of Fig.1.

Table 1. Correction to apply on the yield of the backscattering spectrum of an amorphous silicon target when the detector is at position 2 of Fig.1 instead of position $1\left(2.5 \mathrm{MeV}^{4} \mathrm{He}, \alpha=20^{\circ}\right)$

\begin{tabular}{|c|c|c|}
\hline$\varphi \rightarrow \mathbf{0}^{\circ}$ & Ref. 5 & Eq. 1 \\
\hline $5^{\circ} \rightarrow 0^{\circ}$ & $-3.3 \%$ & $-2.26 \%$ \\
\hline $10^{\circ} \rightarrow 0^{\circ}$ & $-6.7 \%$ & $-4.51 \%$ \\
\hline $15^{\circ} \rightarrow 0^{\circ}$ & $-8.5 \%$ & $-6.79 \%$ \\
\hline
\end{tabular}

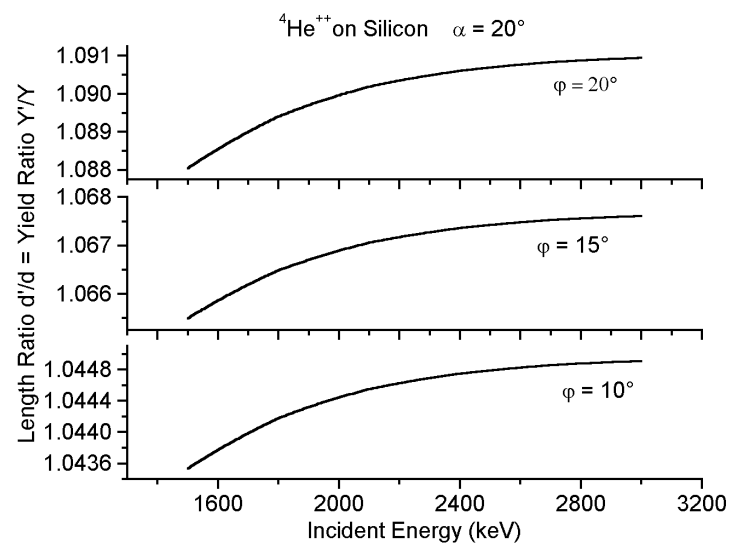

FIGURE 4. The energy dependence of the ratio $d^{\prime} / d=Y^{\prime} / Y$ plotted for $\alpha=20^{\circ}$ and $\varphi=10^{\circ}, 15^{\circ}$, and $20^{\circ}$.

\section{ADDENDUM}

Equation (1) can be generalized for detector positions that are intermediate between the two cases 1 and 2 of Fig. 1. When $\rho$ is defined as an angle that increases in clockwise direction along the arc $\mathrm{C}$ in Fig. 1 from $\rho=0^{\circ}$ at position 1 to $\rho=90^{\circ}$ at position 2 , the yield ratio $\mathrm{Y}^{\prime} / \mathrm{Y}$ for a detector position at an angle $\rho$ is:

$$
\frac{\mathrm{Y}^{\prime}}{\mathrm{Y}}=\frac{1+\frac{1}{\mathrm{rK}_{\mathrm{M}} \cos \alpha}}{1+\frac{\cos \varphi}{\mathrm{rK}_{\mathrm{M}}[\cos (\alpha-\varphi)-(1-\sin \rho) \sin \alpha \sin \varphi]}}
$$

Figure 5 illustrates this dependence. As can be seen, an accurate positioning of the detector near position 2 at $\rho=90^{\circ}$ is uncritical whereas near position 1 at $\rho=0^{\circ}$ each $10^{\circ}$ of offset causes a rise in the yield ratio of about $1 \%$ in the example chosen.
The geometrical arrangement actually employed for the measurement is not given in references $4 \& 5$, but the agreement seen in Table 1 makes that assumption probable. Eleven months later, Ziegler and Brodsky actually report data for a $<111>$-oriented silicon wafer as a function of tilt angle that can only be obtained when the $<111>$ crystal axis sweeps through the detector as the tilt angle increases [7]. It thus appears that throughout that time period the detector was in position 2 of Fig. 1 on the backscattering system at IBM [8]. Had the detector been placed in position 1 instead, the observed change in the yield with the tilt angle would not have arisen.

To avoid complications of the type discussed above, it is best to position the detector in the preferred positions 1 or 1' shown in Fig. 1 whenever possible.

\section{REFERENCES}

1. Lindhard, J., Mat. Fys. Medd. Dan. Vid. Selsk. 34, no 14 (1965).

2. Gemmell, D.S., Rev. Mod. Phys. 46, 129-227 (1974).

3. Eisen, F.H., and Nicolet, M.-A., Nucl. Inst. Meth. B (to be submitted).

4. Chu, W.-K., Mayer, J.W., and Nicolet, M.-A.. Backscattering Spectrometry, Academic Press, New York, 1978, Table VI and Section 2.2.

5. Ziegler, J.F., and Crowder, B.L., Appl. Phys. Letters 20, 178-179 (1972)

6. Ziegler, J.F., and Crowder, B.L., Appl. Phys. Letters 22, 347 (1973).

7. Ziegler, J.F., and Brodsky, M.H., J. Appl. Phys. 44, 188-196 (1973.

8. That this was indeed so was confirmed by private communication with J.F. Ziegler.

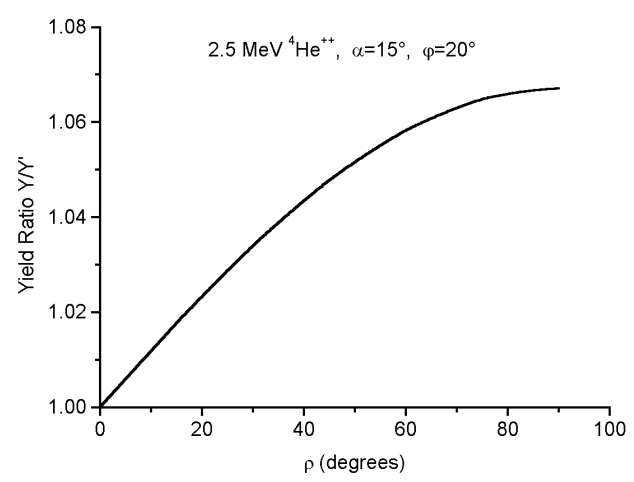

Figure 5. The yield ratio $\mathrm{Y}^{\prime} / \mathrm{Y}$ as a function of the detector position at an angle $\rho$ along the arc $\mathrm{C}$ in Fig. 1 for the case of $2.5 \mathrm{MeV}^{4} \mathrm{He}$ incident on an amorphous silicon target, a detection angle $\alpha=15^{\circ}$, and a tilt angle $\varphi=20^{\circ}$. The angle $\rho=0^{\circ}$ corresponds to position 1 and $\rho=90^{\circ}$ to position 2 in Fig. 1 . 